\title{
La radioprotection pour un cas exceptionnel de radiothérapie peropératoire dans un site non radioprotégé
}

\author{
S. Marcié ${ }^{1 a}$, J. Doyen ${ }^{1}$, M. Tapia ${ }^{2}$, A. Thyss ${ }^{1}$, A. Jeoffray ${ }^{2}$, J.M. Hannoun-Lévi ${ }^{1}$ et J.P. Gérard ${ }^{1}$ \\ 1 Centre Antoine-Lacassagne, 06100 Nice, France. \\ 2 Institut Arnault Tzanck, 06700 Saint-Laurent du Var, France.
}

Reçu le 8 août 2013 - Accepté le 8 décembre 2013

\begin{abstract}
Résumé - Un cas exceptionnel de radiothérapie peropératoire intracardiaque a nécessité l'organisation de ce traitement administrativement, mais aussi en terme de radioprotection. En effet, le traitement devait être effectué dans un établissement non prévu pour des irradiations. Nous avons donc dû préparer, informer, simuler avant la réalisation. Avec l'énergie des photons de $50 \mathrm{kVp}$ et les précautions prises il n'y a eu aucune incidence sur le personnel et l'environnement. Ce cas montre qu'il est possible d'envisager une externalisation de la radiothérapie peropératoire sous réserve des procédures d'autorisation et des mesures de radioprotection.
\end{abstract}

\begin{abstract}
Radiation protection in an exceptional case of intraoperative radiotherapy in a non-radioprotected place. An exceptional case of intracardiac intraoperative radiotherapy required the administrative organization of this treatment, but also in terms of radiation protection. Indeed, the treatment was to be conducted in a facility not designated for radiotherapy, so we had to prepare, inform and simulate before the treatment was carried out. With photon energy of $50 \mathrm{kVp}$ and the precautions taken there was no impact on the staff and the environment. This case shows that it is possible to consider outsourcing of IORT subject to authorization procedures and radiation protection measures.
\end{abstract}

Keywords: radiotherapy / cancer / photons / radiation protection

\section{Introduction}

Un patient de 37 ans présentant une récidive locale précoce au niveau de l'oreillette droite d'un angiosarcome du cœur a nécessité une intervention d'exérèse associée à une radiothérapie peropératoire. Pour cela, il a fallu rassembler les équipes de chirurgie cardiaque et de radiothérapie, ces équipes étant dans des établissements de statuts différents et distants de $12 \mathrm{Km}$. Il fallait aussi intervenir rapidement pour éviter le développement de cette récidive. La procédure de cette réalisation thérapeutique est décrite ci-après.

\section{Le cas clinique}

\subsection{Les angiosarcomes cardiaques}

Le sarcome cardiaque est une entité rarissime dont la prévalence varie entre 0,001 et $0,03 \%$ dans les tumeurs primitives cardiaques malignes (Agaimy et al., 2012). Il survient le plus fréquemment chez des hommes jeunes entre 30 et 40 ans, au niveau de l'oreillette droite. Il n'existait qu'une cinquantaine de cas publiés dans la littérature avant qu'une récente série

\footnotetext{
a serge.marcie@nice.unicancer.fr
}

française de 124 patients soit récemment rapportée et ait permis de faire des avancées considérables dans la connaissance de cette pathologie (Isambert et al., 2013) :

- le pronostic de cette maladie est très péjoratif avec une survie à 5 ans d'environ $20 \%$ en cas d'absence de métastase, et d'environ $0 \%$ en cas de métastases au diagnostic;

- le type histologique est un facteur pronostic majeur et l'angiosarcome correspond au sous-type de plus mauvais pronostic;

- les patients opérés vivent plus longtemps que les patients non opérés et la qualité de la résection doit être macroscopiquement et microscopiquement saine (R0) avec semblet-il une nette supériorité sur la résection microscopiquement non saine (R1) et la résection macroscopiquement non saine (R2);

- en cas de maladie non métastatique la radiothérapie et la chimiothérapie adjuvante permettent de prolonger la survie sans progression, mais pas la survie globale, alors qu'en cas de maladie métastatique la chimiothérapie améliore la survie globale.

En ce qui concerne la radiothérapie, il n'y a aucun consensus sur la dose à délivrer, le volume d'irradiation, l'association à une chimiothérapie ou non. Dans cette série la dose médiane délivrée était de 50 Gy et était donc la dose associée à 

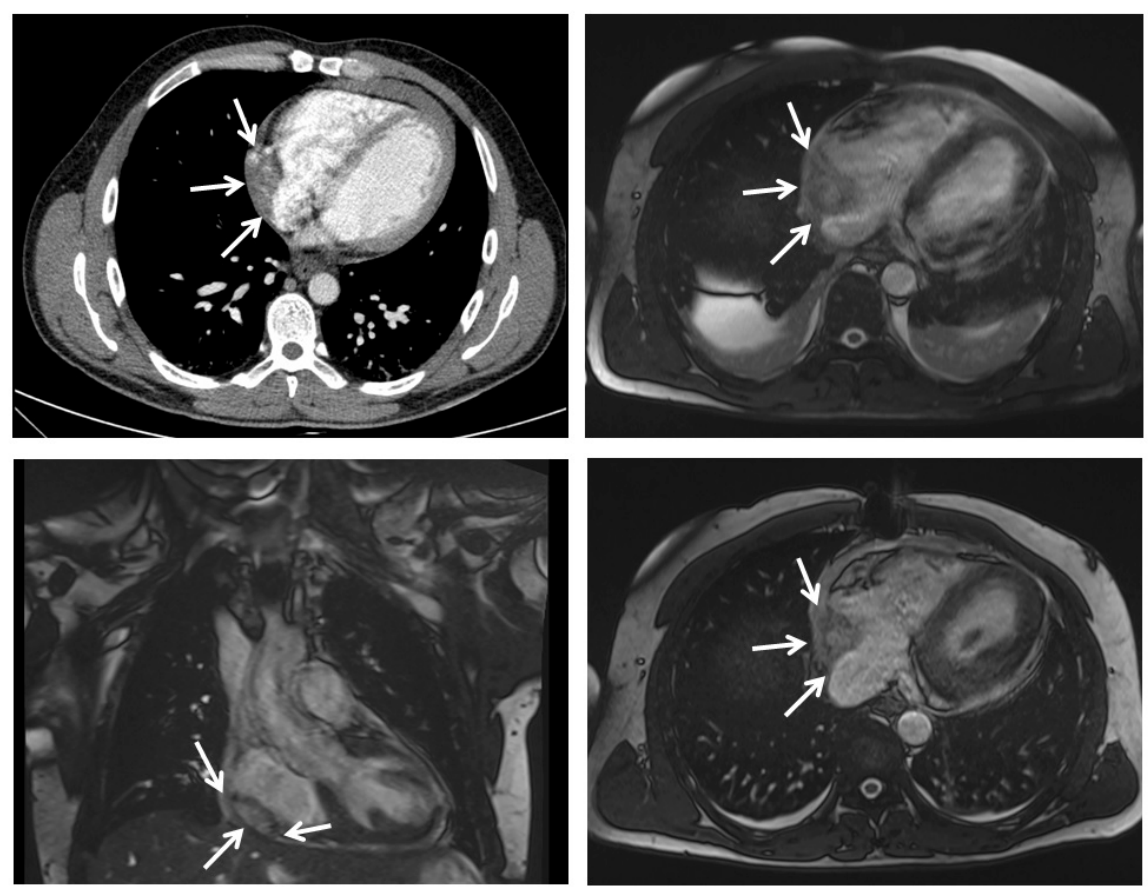

Fig. 1. Imagerie montrant la tumeur sarcomateuse (flèches blanches) au diagnostic (haut gauche, coupe axiale scannographique, haut droit, coupe axiale par imagerie résonance magnétique = IRM séquence T1), et avant la seconde chirurgie et la radiothérapie peropératoire par contact-thérapie (bas gauche, coupe IRM coronale, bas droit, coupe IRM axiale, séquences T1) avec injection de produit de contraste (iode pour le scanner, gadolinium pour l'IRM).

Pictures showing the sarcomatous tumor (white arrows) at diagnosis (top left, axial CT scan, upper right, axial magnetic resonance imaging MRI sequence $=\mathrm{T} 1$ ), and before the second surgery and intraoperative radiotherapy by contact therapy (bottom left, cutting MRI coronal bottom right, axial MRI T1 sequences) with injection of contrast material (iodine for CT scan, gadolinium for MRI).

une meilleure survie sans progression. Elle pourrait être considérée comme la dose minimale à délivrer. Par analogie aux autres sarcomes, en cas de résection R1, il convient de délivrer des doses d'environ 60-66 Gy, et en cas de résection R2, plus de 70 Gy. Il apparait inconcevable d'irradier directement un cœur alors qu'il s'agit d'un organe à risque en temps normal. Les risques d'une telle irradiation n'ont pas ou très peu été précisément rapportés, que ce soit à court ou long terme. Il ne semble pas y avoir de sur toxicités aigues létales d'après la série française, avec un bénéfice qui dépasse le risque eu égard au bénéfice apporté en terme de survie. Les risques potentiels étant la péricardite aigue/chronique, la fibrose myocardique avec trouble de la compliance, des troubles potentiels du rythme cardiaque par atteinte des noeuds de Keith et Flack ou d'Aschoff-Tawara et de leurs branches. La sténose coronarienne avec ischémie d'aval est aussi un risque majeur, qui se démultiplie à chaque Gray reçue en moyenne par le coeur (Darby et al., 2013).

\subsection{Cas clinique}

Il s'agit du cas d'un patient âgé de 37 ans ayant présenté un tableau clinique de tamponnade sur hémorragie péricardique en janvier 2013. L'analyse par imagerie a montré la présence d'une masse de l'oreillette droite (Fig. 1) opérée en urgence, correspondant à un angiosarcome cardiaque de grade 2 de la classification de la FNCLCC (Fédération nationale des centres de lutte contre le cancer (Guillou et al., 1997)) avec marges de résections microscopiquement non saines (R1). Alors qu'une radiothérapie adjuvante était programmée pour février 2013 une rechute locale précoce a eu lieu. Elle s'est manifestée par une douleur thoracique et a été confirmée par l'imagerie par IRM. Une réintervention chirurgicale a donc eu lieu en mars 2013 afin de réséquer en totalité la masse tumorale. Étant donné les difficultés à obtenir une résection R0 dans les chirurgies cardiaques, une radiothérapie peropératoire (RPO) par contact-thérapie avec photons $\mathrm{X}$ de faible énergie $(50 \mathrm{kV})$ a été envisagée. Elle a l'avantage par rapport à une radiothérapie externe avec des faisceaux de haute énergie, de pouvoir délivrer des fortes doses d'irradiation sans irradier de manière excessive les tissus adjacents, avec même une absence quasi-totale d'irradiation à $3 \mathrm{~cm}$ en profondeur ( $c f$. Sect. 6). Dans une série américaine de 18 patients, 2 patients ont été traités par RPO par électrons à hauteur de 5 et 15 Gy en une fraction, qui a l'inconvénient d'entrainer des irradiations ionisantes beaucoup plus en profondeur (Look Hong et al., 2012). Afin d'anticiper une résection $\mathrm{R} 1$ lors de la $2^{\mathrm{e}}$ intervention et de délivrer une radiothérapie le plus précocement possible avec la masse tumorale la plus faible, une contact-thérapie peropératoire a été décidée à hauteur de 7 Gy sur 2 cercles de $3 \mathrm{~cm}$ afin de couvrir tout le lit tumoral. Quelques semaines après la chirurgie le patient a malheureusement rapidement développé des métastases osseuses et une récidive péricardique hémorragique pour lesquelles une 
chimiothérapie par paclitaxel a été débutée, alors qu'une radiothérapie externe était envisagée sur le cœur (mais qui a été annulée). Le patient est toujours vivant aux dernières nouvelles avec une maladie stable.

\section{La préparation}

La décision de la réunion de concertation pluridisciplinaire (RCP) d'effectuer une chirurgie et une radiothérapie peropératoire a justifié l'utilisation des rayonnements ionisants (Article L1333-1 du CSP). Compte tenu de l'urgence, une date a été fixée soit 16 jours après. Les 2 établissements concernés, d'un côté le service de chirurgie cardiaque d'un établissement privé participant au service public et la PCR de l'établissement où l'opération devait s'effectuer, de l'autre le service de radiothérapie d'un centre de lutte contre le cancer qui fournissait le personnel et l'appareil de traitement ont préparé les dossiers d'autorisation pour l'ARS (Agence régionale de santé) et l'ASN (Autorité de sûreté nucléaire).

\section{Les locaux}

Le dossier de radioprotection était spécifique au traitement considéré. Outre les plans de l'étage du bloc opératoire et des niveaux au-dessus (plafond de $30 \mathrm{~cm}$ de béton soit $7 \mathrm{CDA}$ ) et au-dessous (vide sanitaire) le plan de zonage de l'étage a été fourni (Fig. 2). La salle chirurgicale où devait se dérouler la RPO n'est pas radioprotégée (salle 2), avec des cloisons simples, mais avec des dimensions très grandes $\left(8,2 \times 7,8 \mathrm{~m}^{2}\right)$. L'exposition ne pouvant provenir que du rayonnement diffusé, les zones contrôlée et surveillée sont limitées autour de l'appareil $(1 \mathrm{~m})$.

Néanmoins, dans un environnement non habitué aux rayonnements ionisants, nous avons défini une zone interdite autour du bloc.

\section{Le personnel}

Au point de vue personnel, il n'était prévu que le radiothérapeute et le physicien médical présents pendant le traitement (tous les autres intervenants se sont éloignés à l'autre bout du hall, près de la salle 1). Le radiothérapeute disposait des EPI (équipements de protection individuelle : tablier de $0,5 \mathrm{~mm}$ de plomb, lunette et gants stériles et radio-atténuateurs). Le physicien, au pupitre de l'appareil, était à 2 mètres derrière un paravent constitué de $4 \mathrm{~mm}$ de plomb (fourni avec l'appareil).

Pour les mesures, outre le dosimètre passif utilisant la radiophotoluminescence et le dosimètre opérationnel portés par les 2 personnes dans la salle d'opération (pour le radiothérapeute sous le tablier plombé), des dosimètres thermoluminescents de fluorure de lithium (GR200A) ont été disposés en différents endroits (arrière du tube, front du radiothérapeute et sous les gants et le tablier plombés, pupitre, porte d'accès et au niveau de la chaîne).

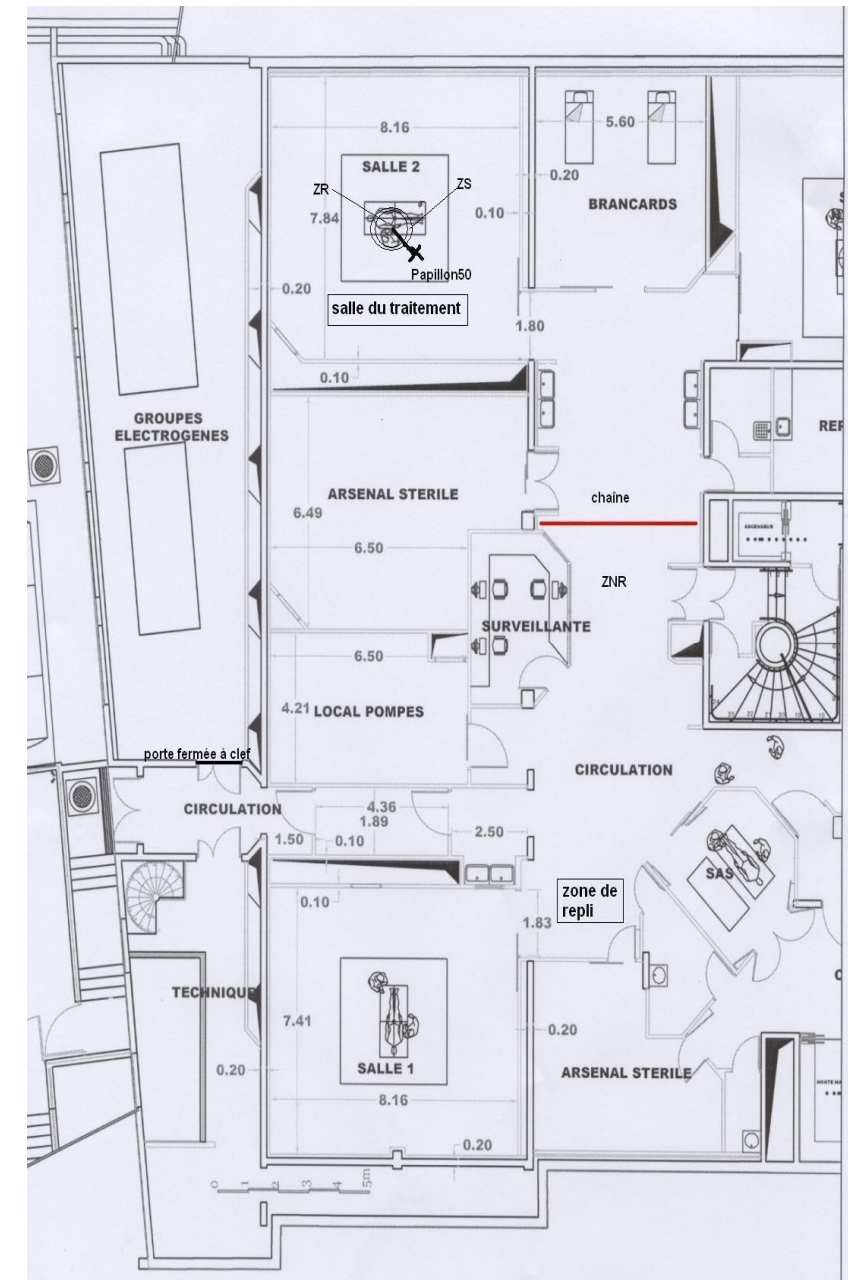

Fig. 2. Le plan du niveau du bloc chirurgical. Map of the surgical block.

\section{L'appareil de traitement}

L'appareil de traitement, «Papillon50 ${ }^{\mathrm{TM}}$ » (Gérard et al., 2012), produit des photons de $50 \mathrm{kVp}(3 \mathrm{~mA}$, CDA 0,7 mm d'Al) avec un débit de $17 \mathrm{~Gy} / \mathrm{min}$ (applicateur diamètre $30 \mathrm{~mm}$ ) à la sortie de l'applicateur (DSP de $32 \mathrm{~mm}$ ). Dans les tissus, la dose décroit rapidement et il ne reste plus que $50 \%$ de la dose à $6 \mathrm{~mm}$ de profondeur (Fig. 3).

Cet appareil n'a pas de pupitre de commande déporté comme cela est exigé pour la RPO de sein (ASN, 2013).

\section{Le traitement}

\subsection{La simulation}

Deux jours avant l'intervention, une simulation a eu lieu dans le bloc opératoire pour permettre au chirurgien et à tout le personnel de se familiariser avec l'appareil. Elle a aussi permis de positionner l'appareil compte tenu de son encombrement et de la nécessité de séparer l'appareil de traitement non stérile (applicateur et tube de rayons $\mathrm{X}$ protégés par une enveloppe en plastique stérile, Fig. 4) et la zone opératoire stérile. 


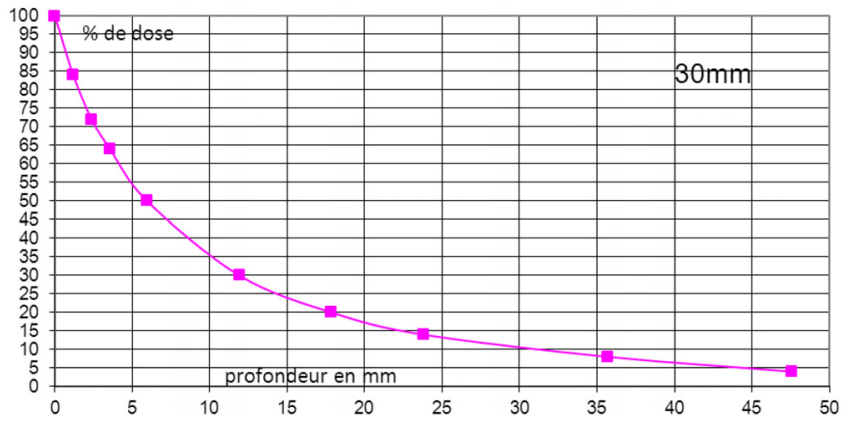

Fig. 3. Courbe de rendement en profondeur dans l'eau pour un applicateur de $30 \mathrm{~mm}$ de diamètre.

Depth dose curve into the water for a 30-mm-diameter applicator.

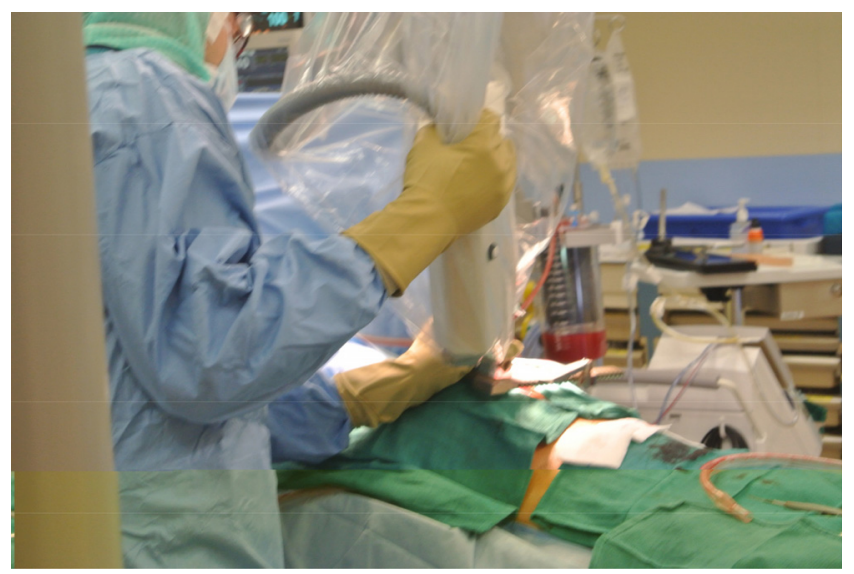

Fig. 4. Le radiothérapeute pendant le traitement maintenant le corps de l'appareil (générateur d'X et le tube avec applicateur). Les mains sont protégées par des gants radio atténuateurs. Le tablier plombé ne se voit pas sous la tenue stérile.

The radiotherapist during the treatment holding the body of the device ( $\mathrm{X}$ generator and tube with applicator). Hands are protected by radioprotective gloves. The lead apron is not visible under the sterile outfit.

\subsection{Le traitement et les doses}

Après l'exérèse de la récidive tumorale, le chirurgien indiquait l'endroit à traiter puis quittait, avec tout le reste du personnel, la salle. Le radiothérapeute, auprès du patient, maintenait le tube de rayons $\mathrm{X}$ et l'applicateur au contact du cœur (Fig. 4).

Comme 2 fois 7 Gy (dose en surface) ont été délivrés, il y a eu moins de 1 minute d'irradiation. Les valeurs données par les dosimètres opérationnels étaient de $1,3 \mu \mathrm{Sv}$. Pour les passifs, les doses étaient inférieures au seuil de détection ainsi que pour les dosimètres thermoluminescents, excepté ceux au niveau du front du radiothérapeute $(30 \mu \mathrm{Sv})$, zone non protégée. Avec la Babyline81 (type E793), la dose mesurée à ce niveau était de $17 \mu \mathrm{Sv}$. Ces résultats sont conformes à ce qui était attendu.

\section{Conclusion}

De manière générale, il apparaît que la RPO utilisant des photons X de moins de $100 \mathrm{kV}$ pourrait permettre d'irradier à de très fortes doses, sans toxicité, des zones que le chirurgien ne peut réséquer. Compte tenu de la maniabilité et de la petite taille de l'appareil de nombreuses zones anatomiques pourraient être traitées comme le cœur. Il faut toutefois faire attention à la dose de prescription qui ne doit pas excéder la contrainte de dose.

Cette technique parait donc une option intéressante à proposer, sans risque sanitaire ou environnemental significatif, avec l'obligation de procédures d'autorisation et des mesures de radioprotection strictes et bien codifiées.

\section{Références}

Agaimy A. et al. (2012) Primary and metastatic cardiac sarcomas: a 12-year experience at a German heart center, Int. J. Clin. Exp. Pathol. 5 (9), 928-938.

Article L1333-1 du code de la santé publique.

ASN (2013) Décision N²013-DC-03459 fixant les règles techniques minimales auxquelles doivent répondre les installations dans lesquelles sont présents des rayonnements $\mathrm{X}$ produits par des appareils fonctionnant sous une haute tension inférieure ou égale à $600 \mathrm{kV}$.

Darby S.C. et al. (2013) Risk of ischemic heart disease in women after radiotherapy for breast cancer, N. Engl. J. Med. 368 (11), 987-998.

Gérard J.P. et al. (2012) Développement de l'appareil Papillon50 ${ }^{\mathrm{TM}}$ et de ses applicateurs pour la radiothérapie $50 \mathrm{kV}$ des cancers du rectum et de la peau, IRBM 33, 109-116.

Guillou L. et al. (1997) Comparative study of the National Cancer Institute and French Federation of Cancer Centers Sarcoma Group grading systems in a population of 410 adult patients with soft tissue sarcoma, J. Clin. Oncol. 15 (1), 350-362.

Isambert N. et al. (2013) Primary cardiac sarcomas: A retrospective study of the French Sarcoma Group, Eur. J. Cancer, DOI: $10.1016 /$ j.ejca.2013.09.012.

Look Hong et al. (2012) Cardiac angiosarcoma management and outcomes: 20-year single-institution experience, Ann. Surg. Oncol. 19 (8), 2707-2715.

Cite this article as: S. Marcié, J. Doyen, M. Tapia, A. Thyss, A. Jeoffray, J.M. Hannoun-Lévi, J.P. Gérard. La radioprotection pour un cas exceptionnel de radiothérapie peropératoire dans un site non radioprotégé. Radioprotection 49(2), 135-138 (2014). 